\title{
Antike Vorstellungen vom Hydrocephalus
}

\author{
Von Ekkehard Pandel*
}

Erst in jüngster Zeit ist durch die neurochirurgische Behandlungsmethode der Shunt-Operation eine aussichtsreiche Therapie des kindlichen Hydrocephalus möglich geworden. Es mag deswegen überraschen, daß Angaben über Entstehung, Art und vor allem Behandlung kindlicher Wasserköpfe bereits bei antiken Autoren zu finden sind. Um zu untersuchen, ob diese Angaben realistisch und (aus unserer Sicht) durchführbar erscheinen oder ob utopische Behandlungsvorschläge gemacht wurden, versuchen wir eine Sichtung der antiken Vorstellungen über den kindlichen Hydrocephalus.

Es finden sich Angaben zu diesem Problem bei Celsus ${ }^{1}$, Pseudo-Galen ${ }^{2}$, PseudoSoran $^{3}$, Aetios ${ }^{4}$, Oreibasios ${ }^{5}$ und Paulos von Ägina ${ }^{6}$. Wir dürfen jedoch ältere Quellen annehmen, denn Celsus als frühester der in diesem Zusammenhang zu nennenden Autoren bezieht sich in seiner kurzen Beschreibung des Hydrocephalus ausdrücklich auf ältere griechische Autoren. Der zeitliche Zusammenhang der Autoren ist so zu sehen: Celsus unter Berufung auf griechische Autoren in den ersten Jahren n. Chr., Galen (129-199 n. Chr.), Pseudo-Soran (etwa 2.Jh. n. Chr.), Oreibasios (Mitte des 4.Jh.n. Chr.) unter Verwendung der Angaben von Anthyllos (unsicher, wohl 2.Jh.n. Chr.), Aetios von Amida im späten 5. Jh.n. Chr. unter Verwendung der Angaben des Leonidas von Alexandria (Ende 1.Jh.n. Chr.), und Paulos von Aegina (7.Jh.n. Chr.) ohne Angaben seiner Quellen.

Von den kürzeren, wenig weiterführenden Texten bei Galen, Pseudo-Soran und Celsus abgesehen, kann zunächst bei Oreibasios, Aetios und Paulos eine weitgehend einheitliche Vorstellung der normalen Anatomie des Schädels gefunden werden. Diese sei kurz vorangeschickt, da die pathologischen Veränderungen bei Hydrocephalus vor dem Hintergrund der anatomischen Vorstellungen leichter verständlich sind.

Im Bereich des Kopfes lassen sich in Aufeinanderfolge der Schichten folgende Einzelheiten unterscheiden: die äußere Schicht bildet die Haut; darunter liegt das «Perikranium», etwa der Schädelaponeurose bzw. Galea entsprechend. Be-

* Herrn Professor Dr.phil.F. Kudlien, Institut für Geschichte der Medizin und Pharmazie der Christian-Albrechts-Universität Kiel, danke ich sehr für den Anstoß zu vorliegender Arbeit, vor allem aber für seinen Rat und seine geduldige Diskussion bei allen auftauchenden Fragen und Problemen. 
sonders Aetios betont, daß in dieser Schicht der Schläfenmuskel liegt. Es folgt die Schicht der durch Nähte begrenzten Schädelknochen. Die Formbarkeit und Elastizität des Schädelknochens des Säuglings betonen Oreibasios und Paulos. Unter dem Schädelknochen liegt die Hirnhaut, darunter das Gehirn. Topographische Angaben, die nicht die Schichtenfolge am Kopf, sondern die Lokalisation an der Schädelzirkumferenz betreffen, finden sich bei Aetios und Oreibasios; es werden benannt die Schläfenregion und die Stirn.

Das Wasserkopfleiden ( durch, daß sich irgendwo am Kopf Flüssigkeit ansammelt. Aetios und Paulos vor allem beziehen den Namen auf das wasserähnliche Aussehen dieser Flüssigkeit; Aetios gibt an - und bezieht die bei ihm besonders ausführlich beschriebene mutmaßliche Ätiologie ein -, daß die Qualität dieser Flüssigkeit manchmal trübe

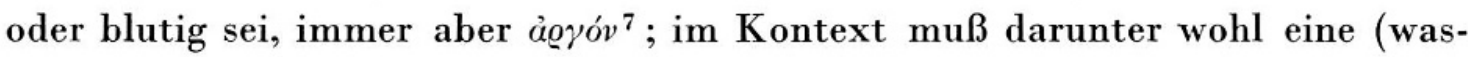
ser-) helle Grundqualität verstanden werden. Bei Paulos findet sich gleichfalls diese Bezeichnung ${ }^{8}$. Aetios nimmt für die Entstehung eines solchen wäßrigen Ergusses am Kopf unbekannte und bekannte Ursachen an: einmal können die Gefäße im Schädelbereich durchlässig werden, und ihr wäßriger Inhalt, mit Blut vermischt, tritt aus; der Grund dafür ist unbekannt. Bekannt ist die Ursache dann, wenn nach einem Schlag oder Stoß Gefäße zerreißen und Blut herausfließt; der dann zunächst blutige Erguß klärt sich später. Zeitbedingte Vorstellungen gehen hier ein : in den Gefäßen befindet sich Blut und eine wäßrige Flüssigkeit; fast mechanisch gedacht wird einmal der wäßrige Bestandteil durch dünn gewordene Gefäße gewissermaßen herausgefiltert; eine grobe Läsion hingegen läßt den gesamten blutig-wäßrigen Inhalt austreten. Der häufigste Grund für eine solche Verletzung, die ätiologisch einen Hydrocephalus bedingt, ist der Druck der Amme gegen den Schädel des Neugeborenen. Eine etwas andere, verschwommenere Vorstellung über das Zustandekommen finden wir bei Paulos; verschwommener deswegen, weil die übergeordnete Ursache allein der Druck der Amme gegen den Kopf eines Kindes sein soll. Erst dann erfolgt die Unterteilung in unbekannte und bekannte Gründe für die Hydrocephalusentstehung. Damit bleibt einmal offenbar unklar, wie und warum die Verletzung durch die Amme zum Hydrocephalus führt; zum zweiten bedingt der Druck eine Gefäßzerreißung mit nachfolgendem Erguß, und drittens werden, offenbar wieder durch die Verletzung, die Gefäße undicht, und der (feuchte) Blutbestandteil wird «ausgeschwitzt». Oreibasios endlich gibt keine ätiologische Deutung; er konstatiert allein, daß ein Hydrocephalus entsteht, wenn die Amme gedankenlos den Kopf des Neugeborenen quetscht. Gemeinsam ist allen die Vorstellung, daß für die Entstehung des Hydrocephalus die rohe Behandlung des Neugeborenenkopfes 
durch die Amme entscheidend ist. Schon hieraus wird deutlich, daß die weiteren Angaben ganz wesentlich die Verhältnisse bei Kindern betreffen. Pseudo-Galen beschreibt die qualitative Zusammensetzung des hydrocephalen Ergusses (wäßrige Flüssigkeit oder getrübtes Blut sammeln sich in einer der den Kopf umhüllenden Schichten); ätiologische Erklärungen finden wir hier nicht.

Entscheidend wichtiger und auf größerem Raum behandelt ist die räumliche Lage der Flüssigkeitsansammlung. In den Details verschieden, lassen sich weitgehend gemeinsame Vorstellungen bei den Autoren finden. Zugrunde liegt dabei die oben gegebene anatomische Einteilung der Schichten am Kopf. Unterscheidbar sind hydrocephale Ergüsse zwischen Haut und Perikranium, zwischen Perikranium und Knochen, zwischen Knochen und Hirnhaut, und zwischen Hirnhaut und Gehirn. Bei Pseudo-Galen werden diese vier möglichen Formen eines Hydrocephalus aufgezählt, jedoch nur schematisch ohne Symptomatik. Aetios teilt ebenfalls in die vier möglichen Formen ein. Bemerkenswert ist zunächst, daß als eine besondere Form der Ergüsse unmittelbar unter der Haut neben der Lage zwischen Haut und Perikranium die Lage zwischen Haut und Schläfenmuskel genannt wird; es bestehen hier besondere Beziehungen zur später erläuterten Therapie. Weitaus wichtiger ist, daß die vierte Form, die Lage zwischen Hirnhaut und Gehirn, zwar genannt, aber in der Einteilung und der späteren Diskussion nicht berücksichtigt wird. Ganz ausdrücklich sagt Aetios, daß die Autoren vor ihm diese vierte Lage beschrieben hätten; es sei jedoch eine Hydrocephalus-

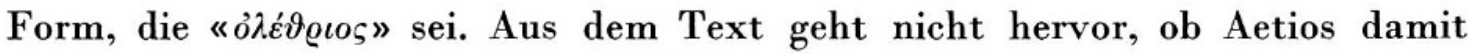
meint, weil Patienten mit dieser Hydrocephalus-Form immer sofort sterben, könne man sie nicht beobachten, oder ob er (wie dann sein ganzer folgender Text zeigt) diese Form aus seiner Erörterung ausklammert, weil er keine Behandlungsmöglichkeit einer sowieso tödlichen Krankheit anbieten kann. Für letzteres könnte folgendes sprechen: Im Zusammenhang mit den Behandlungsmöglichkeiten der vier Hydrocephalus-Formen wird bei Pseudo-Galen ausdrücklich gesagt, es gäbe für den Hydrocephalus zwischen Hirnhaut und Gehirn keine Therapiemöglichkeit. Diese Parallele ermöglicht den Schluß, daß lange vor Aetios die Auffassung bestand, die Hydrocephalus-Form zwischen Hirnhaut und Hirn existiere zwar, sei jedoch unbehandelbar und unweigerlich tödlich. Bei Oreibasios finden wir das gleiche Einteilungsprinzip: Lage zwischen Haut und Perikranium, zwischen Perikranium und Knochen und zwischen Knochen und Hirnhaut; eine besondere Abgrenzung der Lage im Bereich des Schläfenmuskels fehlt. Auch Oreibasios erwähnt die Lokalisation zwischen Hirnhaut und Hirn; seine Auseinandersetzung mit diesem Problem unterscheidet sich jedoch wesentlich von der des Aetios. In starker (bewußter?) Absetzung zu Pseudo-Galen läßt er allein 
die drei zuerst erwähnten Hydrocephalus-Formen gelten. Daß jedoch die vierte Form für ihn wenigstens einer Diskussion wert ist, zeigt seine Argumentation: er meint, diese Form könne nicht beobachtet werden, denn der, der einen Hydrocephalus dieser Art entwickelt, würde sterben, bevor das Leiden manifest wird. Für Oreibasios stellt sich also offensichtlich die Frage einer Behandlungsmöglichkeit gar nicht erst; seine Ablehnung beginnt schon früher. Allerdings fehlt die Begründung für seine Auffassung. Klar liegen die Verhältnisse bei Paulos. Er umgeht die Problematik der vierten Hydrocephalus-Form, indem er nur die drei ersten Lokalisationen aufführt. Die Form zwischen Hirnhaut und Hirn wird mit keinem Wort erwähnt.

Für die weitere Diskussion im Vordergrund stehen also Ergußformen zwischen Haut und Perikranium, zwischen Perikranium und Knochen und zwischen Knochen und Hirnhaut. Wir können an dieser Stelle noch nicht erörtern, ob dieses Einteilungsschema auf pathologisch-anatomischen Kenntnissen basiert. Zuvor muß untersucht werden, wie die einzelnen Hydrocephalus-Formen abgegrenzt werden. Erneut können wir die Diskussion auf die Angaben bei Aetios, Oreibasios und Paulos beschränken; Celsus und Galen geben dazu keine Informationen.

Der Begriff, der Art und Form der hydrocephalen Flüssigkeitsansammlung

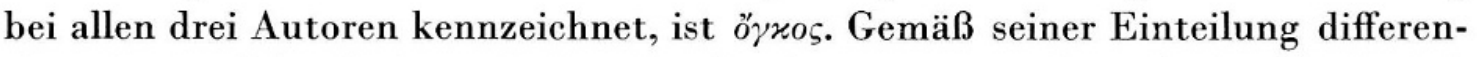
ziert Aetios diese hydrocephalen Geschwülste vorwiegend nach der Ätiologie ${ }^{9}$. Die Geschwulst findet man bei einer Entstehung ohne Ursache von Anfang an, von gleicher Farbe wie die Haut, schmerzlos, gut abgrenzbar und fluktuierend. Differenziert kann davon der Erguß nach einem Schlag werden. Hier bildet sich zunächst eine gerötete und schmerzhafte Geschwulst; später klärt sich die Ergußflüssigkeit, und die Geschwulst gleicht der erstgenannten. Unter Berücksichtigung der Ätiologie (Gefäßzerreißung nach Schlag oder Stoß) erscheint die Betonung von Rötung und Schmerzhaftigkeit verständlich. Wir wollen hervorheben, daß hier eine ätiologische Differenzierung im Vordergrund steht; die Frage der Lokalisation wird nicht diskutiert. Gleichgültig, welcher Lesart man folgt, in jedem Fall scheinen die Symptome der Ergüsse zwischen Haut und Perikranium und Perikranium und Knochen die gleichen zu sein. Wir verstehen hier Aetios wiederum als Pragmatiker, denn, wie wir unten zeigen werden, die Therapie dieser beiden Ergußformen ist gleich; für den Autor erübrigt sich also die Differenzierung.

Wesentlich mehr im Vordergrund steht die Differentialdiagnostik der Ergußlokalisation bei Oreibasios und Paulos. Ein hydrocephaler Erguß unter der Haut ist nach Oreibasios gut abgrenzbar, gleichfarbig, schmerzlos, vorgewölbt, leicht eindrückbar und fluktuierend; eine unter dem Perikranium gelegene Geschwulst 
bietet ganz ähnliche Symptome; die Patienten haben jedoch stärkere Schmerzen; der Erguß ist kleiner und schwerer beweglich, denn es liegen mehr Schichten darüber. Die Beschreibung der Lage zwischen Knochen und Hirnhaut bietet ebenso wie bei Aetios und Paulos besondere Probleme, die unten erörtert werden sollen. Paulos beschreibt einen Erguß unter der Haut mit den gleichen Worten wie Oreibasios; als besonderes Kriterium dieser Ergußform nennt er die leichte Eindrückbarkeit; neben größerer Härte und Schmerzhaftigkeit ist die Ergußform unter dem Perikranium durch eine wegen der dickeren Deckschicht schlechtere Eindrückbarkeit gekennzeichnet. Die Ergußform unter dem Knochen über der Hirnhaut schließlich ist gar nicht mehr eindrückbar. Wir werden die Problematik dieser Ergußform gleich erörtern. Zuvor sollen die diagnostischen Möglichkeiten der zwei ersten hydrocephalen Geschwülste kurz zusammenfassend betrachtet werden. Übereinstimmend findet sich bei der hydrocephalen Flüssigkeitsansammlung eine Geschwulst, die an einer z. B. durch den Schlag bestimmten Stelle des Kopfes lokalisiert ist. Zur weiteren Diagnostik werden Palpation, Inspektion und subjektive Angaben des Patienten verwertet. In jedem Fall ist die Schwellung gut abgrenzbar, elastisch und eindrückbar. Je nach Lage zwischen den Schädelschichten nimmt die Konsistenz zu; besonders Paulos wählt die strenge Dreiteilung auch bei der Differenzierung der Symptomatik. Wir sehen eine recht naturalistische Auffassung: je stärker die Deckschicht ist, je tiefer also der Erguß liegt, desto schwerer ist es, den Finger hineinzudrücken. Neben der subjektiven Schmerzangabe ist insbesondere diese wechselnde Kompressibilität das differentialdiagnostische Kriterium. Da wir an anderer Stelle auf das Problem zurückkommen werden, inwieweit diese Beschreibungen unseren Vorstellungen entsprechenden Krankheitsbildern vergleichbar sind, sei hier nur auf die bereits sinnfällige Problematik hingewiesen: wir stehen vor der Schwierigkeit zu entscheiden, ob einer vorgegebenen anatomischen Einteilung möglicherweise sehr unterschiedliche klinische Befunde untergeordnet und in das vorgesehene Schema gepreßt werden oder ob zunächst klinische Krankheitsbilder beobachtet und dann bekannte anatomische Strukturen zugeordnet werden.

Wesentlich schwieriger als die Interpretation der Angaben zu HydrocephalusFormen zwischen Haut und Perikranium und zwischen Perikranium und Knochen, der extrakraniellen Lokalisation also, ist die Untersuchung der Beschreibung der intrakraniellen Ergüsse zwischen Knochen und Hirnhaut. Die Schwierigkeit liegt insbesondere in der außerordentlichen Unterschiedlichkeit, ja Widersprüchlichkeit der Angaben der einzelnen Autoren im Vergleich zur Einheitlichkeit der bisher untersuchten Beschreibungen.

Aetios unterteilt die zwischen Knochen und Hirnhaut liegenden Ergüsse in 
zwei Formen. Bei kleinen Ergüssen stehen, und das sei schon hier besonders betont, klinische Symptome im Vordergrund: der Kopf des Patienten wird schwer, es treten Ohnmachten auf, und die Sinne stumpfen ab; am Schädel selbst ist in diesem Stadium offenbar keinerlei Veränderung zu beobachten. Erst wenn die Flüssigkeitsmenge zunimmt, zeigen sich äußere Symptome: die Schädelnähte weichen auseinander; damit nimmt der Schädelumfang zu. Ohne unseren weiteren Erörterungen vorzugreifen, sei hier bereits angemerkt, daß diese Symptomatik recht genau einer intrakraniellen Drucksteigerung beim Säugling entspricht. Schwieriger zu interpretieren ist die folgende Angabe: aus Nahtdehiszenz und Größenzunahme werde der Ort der Flüssigkeitsansammlung offensichtlich, denn die Flüssigkeit sei mit dem Finger wegdrückbar. Offenbar muß, ohne daß ein

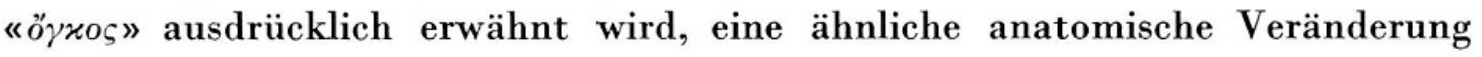
wie bei den ersten beiden Hydrocephalus-Formen angenommen werden. Aetios selbst gibt dann bei der Beschreibung der Therapiemöglichkeiten an, die Flüssigkeit werde bei großen Ergüssen zwischen Knochen und Hirnhaut mit Nahtdehiszenz sichtbar; in welcher Form das geschieht, wird jedoch nicht gesagt.

Die Entstehung einer Geschwulst auch bei einer Flüssigkeitsansammlung zwischen Knochen und Hirnhaut nehmen ausdrücklich Oreibasios und Paulos an. Vor allem Oreibasios unterscheidet dabei zwei besondere Formen: einmal entsteht durch die Flüssigkeitsansammlung unter dem Knochen eine Vorwölbung, da der kindliche Schädelknochen weich und verformbar ist; es entsteht dabei ein fester, schmerzhafter und spitz ausgezogener Erguß. Bemerkenswert sind die Angaben zu den entsprechenden Veränderungen am Schädel: Oreibasios gibt an, daß Stirn- und Schläfengegenden dabei enger zusammenrücken als normalerweise, da die Strukturen durch die Ausziehung nach oben zusammengedrängt werden. Offenbar muß hierbei eine Lage des Ergusses in der Mittellinie der Scheitelgegend angenommen werden. Aber auch dann ist schwierig zu deuten, welche Vorstellung zugrunde liegt. Wir meinen zwei grundsätzliche Möglichkeiten zu sehen: einmal könnten durch einen nach oben spitz ausgezogenen Schädel die Schädelproportionen so verändert werden, daß vergleichsweise zur Höhenausdehnung die Stirnpartie verschmälert erscheint. Möglicherweise aber liegt auch eine fast geometrische Vorstellung zugrunde: vorausgesetzt, die gesamte Umfangslänge der Schädelknochen bliebe unverändert, dann müßte wie bei der Höhenveränderung eines Dreiecks eine Ausziehung nach oben eine Verkürzung der Basis bedingen. Auf jeden Fall wird eine Verkürzung des Stirn-SchläfenAbstandes angenommen und wohl kausal damit die klinische Symptomatik verknüpft: die Kranken haben feurige Blitze vor den Augen und häufigen Lidschlag ${ }^{10}$. Wir finden also zumindest ein objektiv beobachtbares Symptom im 
Augenbereich; die bei Oreibasios angenommene Ursache dafür ist der Druck auf die Augenregion. Eine zweite Möglichkeit der Auswirkung einer Flüssigkeitsansammlung zwischen Knochen und Hirnhaut schließt sich unmittelbar an:

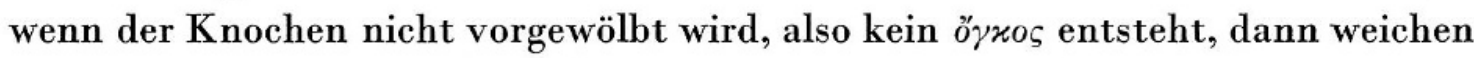
die Schädelnähte auseinander, und durch den Nahtspalt tritt die Flüssigkeit unter das Perikranium, um dann zwischen Knochen und Perikranium zu liegen. Die Symptome sind dann die gleichen wie bei einem primär zwischen Knochen und Perikranium entstandenen Erguß; wohl aufgrund der zusätzlichen Nahtdehiszenz haben die Kranken jedoch stärkere Schmerzen. Dazu kommen noch zwei besonders herausgehobene diagnostische Kriterien: man kann von außen her durch konzentrischen Druck den Erguß verkleinern, so daß der Eindruck entsteht, als weiche ein Teil der Flüssigkeit in das Schädelinnere durch den Nahtspalt hindurch zurück. Schwieriger zu verstehen ist die Beschreibung des zweiten Symptoms: wenn man mit der Hand gleichmäßig über die Ergußgeschwulst fährt, habe man an einer Stelle den Eindruck, als gerate man gleichsam in einen Hohlraum (oĩov $\varkappa \varepsilon v \varepsilon \mu \beta \alpha \tau \varepsilon \check{\imath}$ ); dies sei die Stelle, an der die Nähte dehiszent sind. Offenbar muß die Konsistenz der Ergußgeschwulst also uneinheitlich sein, etwa in der Form, daß eine besonders weiche Stelle von festerer Substanz umgeben ist und so gewissermaßen unter der Palpation wie ein Hohlraum wirkt; eine mögliche Erklärung dafür werden wir später diskutieren. Wir finden bei Oreibasios also die Vorstellung, daß auch bei einer intrakraniellen Lage eine tastbare Geschwulst entsteht: einmal durch die Knochenvorwölbung, bei der der Erguß dann unmittelbar unter dem Knochentumor liegt; dann nach Nahtdehiszenz durch die Verlagerung der Ergußflüssigkeit unter das Perikranium. Wann jeweils eine der beiden Möglichkeiten verwirklicht wird, ob also etwa härtere Knochen beim älteren Kind eher die Nahtdehiszenz bedingen oder ob die Ergußgröße eine Rolle spielt, wird nicht gesagt. Zur Differenzierung der Formen untereinander und gegenüber den primär extrakraniellen Formen werden im wesentlichen morphologische Kriterien herangezogen. Nur einmal wird zur Differenzierung ein nicht morphologisches klinisches Symptom herangezogen.

Die Auffassung, auch bei intrakranieller Lage entstehe eine Geschwulst, vertritt auch Paulos. Im Ansatz finden wir die eben bei Oreibasios herausgearbeitete Zweiteilung der möglichen Formen wieder; die Abgrenzung bei Paulos aber ist weniger scharf. Die entstehende Geschwulst ist gegenüber den extrakraniellen Formen nicht eindrückbar und nicht gut tastbar; sie weicht nur aus, wenn mit großer Kraft dagegen gedrückt wird. Die Entstehung der Geschwulst wird einmal erklärt durch die Weichheit des kindlichen Schädelknochens und dann vor allem durch die Durchlässigkeit der Schädelnähte, die eine Verlagerung des 
Ergusses nach außen ermöglicht. Offenbar allein auf diese letztgenannte Entstehungsart bezogen, werden dafür diagnostische Kriterien genannt: bei kräftigerem Druck läßt sich die Ergußflüssigkeit in die Tiefe (des Schädels) zurückdrängen. Eine gewisse Schwierigkeit der Interpretation liegt in der gedanklichen Verknüpfung. Nachdem Paulos zunächst die Entstehung einer Geschwulst auch bei intrakranieller Ergußlage annimmt und beschreibt, folgt in kausaler Verknüpfung die Angabe, der Knochen sei weich und die Schädelnähte durchlässig. Man kann zunächst den Eindruck gewinnen, beide Symptome seien Hinweis auf eine gemeinsame Ursache und Ausdruck einer Krankheitseinheit, bei der eben diese Symptome nur zusammen auftreten. Unter Berücksichtigung des Oreibasios-Textes können damit aber auch zwei grundsätzlich mögliche Entstehungsmechanismen der Geschwulst bei intrakraniellem Erguß gemeint sein; die Vorstellung wäre dann allerdings weniger genau formuliert als bei Oreibasios. Wir neigen dazu, diese letzte Möglichkeit anzunehmen. Die Angabe (S.46, Z.28), die Flüssigkeit sei in die Tiefe drückbar, bezöge sich dann allein auf die Form, bei der eine Verlagerung durch die Schädelnähte nach außen erfolgte. Ebenfalls darauf bezogen wäre dann die im weiteren (S.47, Z.1ff.) geschilderte Symptomatik: die Kranken haben stärkere Schmerzen; der Schädel weicht auseinander; sie blicken starr und weinen häufig. Die Geschwulst selbst wird dabei kaum berücksichtigt; eine Qualitätsbeschreibung fehlt. Ganz im Vordergrund stehen die Auswirkungen. Wenn wir die Angaben bei Oreibasios und Paulos vergleichen, fällt vor allem diese Verlagerung des Schwerpunktes auf. Da bei Paulos an keiner Stelle gesagt wird, der Knochen werde durch den Erguß vorgewölbt - eine Angabe, die wir expressis verbis bei Oreibasios finden -, mag auch von der Ätiologie her der Schwerpunkt auf einer Entstehung der Geschwulst durch Nahtdehiszenz und Verlagerung der Flüssigkeit nach außen liegen.

Wir haben bisher eine Fülle von Einzelangaben betrachtet, die alle zur näheren Erläuterung des Begriffes «Hydrocephalus» verwandt werden. Das Hauptproblem liegt nun vor allem darin, zu untersuchen, ob auf der Grundlage unserer Kenntnisse und Konzepte Bezüge zwischen den antiken Beschreibungen und heute definierten Krankheitsbildern bestehen. Wir deuteten bereits oben an, daß uns grundsätzlich zwei Ansatzpunkte möglich erscheinen. Einmal könnten pathologische Zustände beschrieben worden sein, die wir auch heute ähnlich beschreiben würden, wenn auch unter anderen Krankheitsbegriffen. Andererseits könnte der Beschreibung eine so grundlegend andere Vorstellung über Art und Wesen einer Krankheit zugrunde liegen und die Beschreibung eines beobachteten pathologischen Zustandes so auf dieses Krankheitskonzept zugeschnitten sein, daß es nur schwer oder gar nicht möglich wäre, heute gebräuchliche Krankheitsbegriffe 
aus den Texten wiederzuerkennen. Sicherlich werden in diesem Zusammenhang Probleme berührt, die über die inhaltliche Bedeutung der hier behandelten Texte hinausgehen. Wir wollen dennoch versuchen, darzustellen, was die antiken Autoren mit ihren Angaben im Vergleich zu unseren Krankheitsvorstellungen gemeint haben können.

Wir fanden bisher im wesentlichen übereinstimmende Angaben bei der Beschreibung von Flüssigkeitsansammlungen außerhalb der knöchernen Schädelkapsel. Abgesehen von den Angaben bei Celsus, der in seiner sehr kurzen Textangabe einen Hydrocephalus als chronische Flüssigkeitsdurchtränkung der Haut des Schädels bezeichnet, beschreiben alle Autoren die extrakraniellen Geschwülste als gut abgrenzbare, tastbare Tumoren; weitere hervorgehobene Qualitäten sind Schmerzlosigkeit, Eindrückbarkeit und Fluktuation. Grundsätzlich sind diese Kriterien für die beiden möglichen Formen der extrakraniellen hydrocephalen Ergüsse gleich; eine Differenzierung wird dann auf eher quantitativem Wege versucht: tiefer gelegene Ergüsse unter dem Perikranium bereiten eher Schmerzen, sind weniger gut eindrückbar und so härter. Wenn wir Krankheitsbilder suchen, die den gegebenen Beschreibungen entsprechen können, so findet sich einiges in der pädiatrischen Krankheitslehre, nämlich die traumatischen Geschwülste beim Neugeborenen und die angeborenen Celen im Kopfbereich. Wir wollen zunächst die allein bei Aetios beschriebene akut entstandene traumatische Form hinzunehmen. Rötung und Schmerzhaftigkeit kennzeichnen möglicherweise einen entzündlichen Prozeß; denkbar ist jedoch auch ein einfaches subkutanes Hämatom; der weitere Verlauf, die von Aetios angenommene «Klärung» zum geläufigeren, nicht akut entstandenen Erguß allerdings läßt sich in der angegebenen Form nicht nachvollziehen. Möglicherweise werden hier zwei getrennte Beobachtungen aus einem Kausalitätsbedürfnis heraus miteinander verknüpft. An für die weitere Diskussion in Frage kommenden fluktuierenden Geschwülsten am Schädel des Neugeborenen nennen wir die traumatischen Geburtsgeschwülste (Caput succedaneum und Cephalhämatom) und die verschiedenen Formen der angeborenen Cephalocelen (Meningocelen, Meningoencephalocelen). Wir dürfen annehmen, daß die gleichen uns bekannten Krankheitsbilder im Prinzip bereits früh vorgekommen sind, da die Entstehungsursachen (angeborene Knochendefekte, mechanische Alteration im Geburtskanal) keine grundsätzlichen Veränderungen erfahren haben. Das Caput succedaneum als druckbedingte ödematöse Durchtränkung des Gewebes zwischen Haut und Galea entspricht in den Qualitäten Farbe, Konsistenz und weitgehende Schmerzlosigkeit gut den beschriebenen Kopfgeschwülsten; es fehlen allerdings die gute Abgrenzbarkeit und vor allem die Chronizität. Zwar vermißten wir, abgesehen von Celsus, 
der ausdrücklich eine chronische Verlaufsform annimmt, in allen Fällen eine Zeitangabe; uns scheint aber ein Verlauf, der über das in der Regel nicht länger als zwei Tage dauernde Bestehen des Caput succedaneum hinausgeht, in die jeweiligen Angaben der Autoren eingeschlossen. Sicherlich über längere Zeit jedoch bestehen Cephalhämatome und Cephalocelen. In jedem Fall genügen beide Geschwulstformen den angegebenen Kriterien. Das tiefgelegene, von der Haut, der Galea und dem Periost bedeckte Cephalhämatom bildet in aller Regel einen prallelastischen, gut abgrenzbaren, hautfarbenen Tumor, der praktisch schmerzlos ist. Ganz ähnlich ist die Morphologie der meist in der Medianlinie gelegenen Cephalocelen. Bei diesen treten Teile des Schädelinhalts (Hirnhaut mit Liquor; Hirnhaut, Liquor und Hirnteile) durch angeborene Knochendefekte aus und sind unter der Haut tastbar. Auch heute ist es außerordentlich schwierig, allein durch Inspektion und Palpation im Einzelfall zu entscheiden, welche Form von Tumor vorliegt; in der Regel bringt erst die Röntgenuntersuchung Klarheit. Da jedoch Cephalhämatome ungleich häufiger sind als Cephalocelen, dürfen wir vermuten, daß die beschriebenen Tumorformen in den meisten Fällen Cephalhämatome gewesen sind; beide Tumorformen können jedenfalls in der Weise beschrieben werden, die wir in den Texten finden. Eine weitere Differenzierung allerdings dürfte nicht möglich gewesen sein.

Sehr viel schwieriger ist die Übertragung der beschriebenen Vorstellungen über die intrakraniellen Flüssigkeitsansammlungen. Wir haben bereits bei der Einzelanalyse betont, wie stark vergleichsweise Unterschiede in der Beschreibung sind. Dabei ließen sich zwei grundsätzliche Kategorien der Symptomatik unterscheiden: einmal morphologische Kriterien, dann eher klinische Symptome. Wir beginnen mit den Angaben zur Morphologie.

Wir fanden, daß Oreibasios und Paulos ausdrücklich auch bei Lage des Ergusses unter dem Schädelknochen eine nach außen vorgewölbte Geschwulstbildung beschreiben. Beide nehmen offenbar an, daß durch den Druck der Flüssigkeit der weiche kindliche Knochen vorgewölbt werden kann und dadurch ein sehr harter, nicht fluktuierender Tumor entsteht. Wir können nur vermuten, daß mit dieser Beschreibung ein verkalktes Cephalhämatom gemeint ist. Zumindest kann nach Inspektion und Palpation ein solcher «Knochentumor» wie eine durch Druck von ihnen verursachte Knochenvorwölbung wirken. Auf die zusätzlich bei Oreibasios beschriebene problematische «Verengung» der Stirnpartie sind wir bereits eingegangen.

Wir fanden dann die Beschreibung einer zweiten Form; hierbei soll durch Nahtdehiszenz die Ergußflüssigkeit an die Außenseite des Schädelknochens gelangen und dort eine tastbare Geschwulst bilden. Vage bleibt die Beschreibung 
bei Aetios: nach Nahtdehiszenz wird die Stelle der Flüssigkeitsansammlung deutlich; bis auf die Angabe, die Flüssigkeit lasse sich mit dem Finger eindrücken, fehlen Beschreibungen der Morphologie. Jedoch dürfen wir aus seinen Aussagen auf doch wohl tastbar erweiterte Schädelnähte, Kopfumfangserweiterung und palpable Vorwölbung schließen. Genauer beschreiben Oreibasios und Paulos die Art der Geschwulst: die ähnlich wie bei extrakranieller Lage tastbare Vorwölbung läßt sich mit größerer Kraft durch die erweiterten Schädelnähte zurück in das Schädelinnere drängen. Wir haben die Einzelheiten oben beschrieben. Uns fehlt eine genauere Angabe über den Sitz der Geschwulst an der Schädelzirkumferenz; aber wir möchten die Angaben interpretieren als eine Beschreibung einer Dehiszenz der Schädelnähte mit Vorwölbung der Fontanelle. Schwierig ist in diesem Zusammenhang das bei Oreibasios angegebene Kriterium zu deuten, bei Palpation der Geschwulst taste man an einer Stelle gleichsam eine Höhlung. Ein vergleichbares Krankheitsbild nur aus dieser Angabe zu finden, fällt schwer. Aber wenn wir allein die Untersuchungsmethode der Palpation zugrunde legen, dann kann u. U. ein von der Peripherie her zirkulär verknöcherndes Cephalhämatom ein so weiches Zentrum haben, daß der Eindruck einer unter der Haut gelegenen Höhlung entsteht. Es bliebe dann zu klären, wie diese Höhlung entstanden ist. Bei Oreibasios finden wir eine Deutung: die Höhlung stehe in Beziehung zur Nahtdehiszenz. In offensichtlich sehr mechanischer Denkweise wird also wohl angenommen, Substanz des Ergusses fließe durch die Nahtdehiszenz ab und ein Hohlraum bleibe zurück. Es ist jedoch nur schwer möglich, eine beginnende Verknöcherung eines Cephalhämatoms mit einer Nahtdehiszenz zu verbinden. Allerdings müssen wir bei dieser Gelegenheit betonen, wie ungenau und teilweise widersprüchlich die Vorstellungen antiker Autoren über Lage und Verlauf der Schädelnähte waren ${ }^{11}$. An keiner Stelle unserer Texte wird gesagt, wie eine Nahtdehiszenz zu diagnostizieren sei. Zudem sind wohl alle Vorstellungen über Schädelnähte in der Antike an Verhältnissen des knöchernen Leichenschädels gewonnen; Beschreibungen der Verhältnisse am Schädel des lebenden Säuglings sind uns nicht bekannt. Wir müssen so offenlassen, welche Vorstellung der Beschreibung bei Oreibasios zugrunde liegt. Berücksichtigen wir jedoch vor allem die zuvor beschriebenen Kriterien, so gleicht die gegebene Beschreibung (Dehiszenz der Schädelnähte mit Vorwölbung der Fontanelle) bis in die Einzelheiten den morphologischen Befunden bei gesteigertem Schädelinnendruck des Säuglings. Besonders eindrucksvoll ergänzen und stützen diese Auffassung die jeweils beschriebenen und nur bei dieser Form des Ergusses angegebenen klinischen Zeichen : der Kopf wird schwer; es treten Stadien von Bewußtlosigkeit auf; die Sinne stumpfen ab (Aetios); durch starken Druck läßt sich unter starken Schmerzen 
der Tumor verkleinern: tatsächlich kann man im Gegensatz insbesondere zum Cephalhämatom durch starken Druck auf die vorgewölbte Fontanelle erreichen, daß keine Vorwölbung über das Knochenniveau mehr tastbar ist. Und noch einmal vor allem die Angaben bei Paulos: die Kinder haben starke Schmerzen; der Kopf ist aufgetrieben; die Stirnpartie wölbt sich vor; sie blicken starr und weinen viel.

Wir müssen an dieser Stelle kurz einfügen, wie heute der Begriff «Hydrocephalus» verstanden wird. Definitionsgemäß bedeutet er eine Vermehrung der intrakraniellen Liquormenge mit Steigerung des intrakraniellen Druckes unter Schädigung der Hirnsubstanz; alle extrakraniellen und insbesondere alle umschriebenen intrakraniellen Flüssigkeitsansammlungen (z. B. epidurales Hämatom, subdurales Hämatom, Hygrome) - fallen in Einengung des Hydrocephalus-Begriffes nicht mehr darunter. Andererseits aber ist ein Hydrocephalus im engeren Sinne nur eine Form einer intrakraniellen Drucksteigerung. Bei Säuglingen müssen bei entsprechender Symptomatik differentialdiagnostisch alle anderen raumfordernden intrakraniellen Prozesse (Tumoren, Blutungen) bedacht werden. Bemerkenswert erscheint uns jedoch, daß eine genaue Analyse der antiken Texte doch eine recht genaue Schilderung der Symptome einer intrakraniellen Drucksteigerung aufdeckt. Natürlich ist der Ansatzpunkt verschieden: die tastbare vorgewölbte Fontanelle wird als umschriebener intrakranieller Erguß gedeutet; die Nahtdehiszenz ermöglicht die «logische» Erklärung für die Verlagerung der Flüssigkeit über das Knochenniveau. Was wir als sicher annehmen dürfen, ohne die Angaben in der Interpretation überzustrapazieren: intrakraniell drucksteigernde Prozesse sollten auch damals vorgekommen sein; die vergleichsweise exakten, wenn auch bei den einzelnen Autoren in sich zum Teil widersprüchlichen Schilderungen der morphologischen und klinischen Symptomatologie stützen diese Auffassung. Nur kann aus den angegebenen Symptomen allein nie die genaue Ätiologie geklärt werden. In gleicher Weise ist ein Hydrocephalus in heutigem Sinne denkbar, wie auch epidurale Ergüsse oder intrakranielle Tumoren und Blutungen. Es können also auch Hydrocephalus-Formen im heutigen Sinne beschrieben worden sein; generell darf aber aus unserer Sicht höchstens auf ein Krankheitsbild beim Neugeborenen mit intrakranieller Drucksteigerung geschlossen werden. Wenn jedoch aufgrund einer vorgegebenen anatomischen Einteilung allein eine intrakranielle Flüssigkeitsansammlung zwischen Hirnhaut und Knochen für möglich gehalten wird - und das tun Aetios, Oreibasios und Paulos -, dann ermöglichen die angegebenen Kriterien genau diese Diagnose und die Abgrenzung von den extrakraniellen Ergüssen. Da die klinische Symptomatik und die Schädelmorphologie, insbesondere die Stärke der Fontanellenvorwölbung, mehr als der morphologische 
Befund eines Cephalhämatoms vom Ausmaß des Prozesses abhängen, mag sich daraus die größere Unsicherheit und teilweise Widersprüchlichkeit in der Beschreibung erklären. Wir möchten jedoch annehmen, daß tatsächlich klinische Beobachtungen beschrieben werden; die gemeinsame Betrachtung aller Angaben bietet dafür genügend Belege. Eine weitere Stützung erfährt diese Auffassung durch eine Betrachtung der vorgeschlagenen Therapien. Sie sind durchweg chirurgisch, zeigen jedoch interessante Modifizierungen bei den Autoren.

Wir nehmen wiederum Angaben bei Celsus und Pseudo-Galen vorweg. Beide beschreiben nur kurz ohne wesentliche Erklärungen. Celsus gibt als Therapie seines als Hydrocephalus bezeichneten Krankheitsbildes an, der Schädel sei zu rasieren, danach ein Senfpflaster aufzulegen, bis die Haut ulzeriere; falls das nichts nütze, müsse mit dem Skalpell eingeschnitten werden. Zusätzlich werden allgemeine Maßnahmen zur «Entwässerung» angegeben: körperliche Übung, Schwitzen, Diurese. Da aus Celsus' wenigen Angaben nicht sicher hervorgeht, welches Krankheitsbild gemeint ist - möglich sind Ödeme des extrakraniellen Gewebes, Caput succedaneum oder Cephalhämatom -, kann auch über Erfolg oder Gefahren der angegebenen Therapie wenig mehr gesagt werden. PseudoGalen (Bd.14, S.783, Z.4-7) gibt zur Behandlung der hydrocephalen Ergüsse unter der Haut und unter dem Perikranium an, die Geschwülste seien durch zwei oder drei gerade Einschnitte zu entleeren; Ergüsse unter dem Knochen müßten «herausgeschlagen», also wohl nach Trepanation entleert werden. Ergüsse zwischen Hirnhaut und Hirn seien nicht therapierbar.

Umfangreicher und genauer sind die Behandlungsvorschläge bei Aetios, Oreibasios und Paulos. Weitgehende Übereinstimmung besteht über die Behandlung der extrakraniellen Geschwülste. Behandelt wird chirurgisch mit dem Ziel einer Entleerung des Ergusses. Die Parallele zur Abszeß-Chirurgie liegt nahe und wird in jedem Fall gezogen. Bei kleinen Ergüssen solle ein kleiner Einschnitt auf der Kuppe gemacht werden, bei größeren zwei oder drei parallele Schnitte an den Geschwulsträndern (Aetios); das gleiche Vorgehen gibt Oreibasios an. Beide erwähnen als therapeutisches Problem den subperikranial unter dem Schläfenmuskel gelegenen Erguß. Der Muskel sei nicht einzuschneiden, sondern man müsse Schnitte beidseits an den Muskelrändern legen; nach Aetios soll der Muskel dann von der Seite her angehoben und der Erguß entleert werden. Paulos gibt gleiche Vorschläge für die chirurgische Behandlung der unter der Haut gelegenen Ergüsse wie Aetios und Oreibasios; für die unter dem Perikranium gelegenen Ergüsse gibt er eine H-förmige Schnittführung an. Sehr genau und übereinstimmend sind die jeweiligen Angaben zur Verbandstechnik. Besonders ausführlich beschreibt Aetios Tamponade, Charpieauflagen und wollene Binden als Verbands- 
material. Feste Verbände hält er für Kinder nicht für geeignet, da sie zu schwer sind; es genügen wollene Verbände. Oreibasios gibt keine Einzelheiten an, sondern verweist auf die analoge Behandlung bei Abszessen. Paulos verbindet «in üblicher Weise»; der Verband wird bis zum dritten Tag mit Weinöl befeuchtet. Ebenso wie Aetios gibt er an, daß auf diese Weise bis zum dritten Tag eine Primärheilung erreicht werden kann. Danach genügt dann ein Wundpflaster. Offenbar sind größere Weichteildefekte möglich, denn abschließend schlägt Paulos vor, den Knochen, falls er längere Zeit von Gewebe nicht bedeckt sei, anzuritzen.

Uns scheinen diese übereinstimmenden Angaben und die bis ins Detail auch heutigen chirurgischen Praktiken entsprechenden Vorschläge genügend Hinweise zu geben, daß die angegebenen Behandlungen tatsächlich durchgeführt worden sind. Wir haben oben gesehen, daß die Diagnose einer extrakraniellen Geschwulst möglich war. $\mathrm{Ob}$ die Geschwulst unter der Haut oder unter dem Perikranium liegt, ist dann von untergeordneter Bedeutung. Allerdings sind, wie erwähnt, neben dem Cephalhämatom auch andere Geschwülste, vor allem Cephalocelen, differentialdiagnostisch wichtig. Hier scheint uns eine weitere Differenzierung nicht mehr möglich. Wenn wir jedoch nach unseren heutigen Kenntnissen voraussetzen, daß die bei weitem häufigste Geschwulst des Schädels, die oben genannten Kriterien entspricht, das Cephalhämatom ist, erscheinen Erfolge der beschriebenen Therapie möglich. Es mögen dann auch hin und wieder Encephalocelen inzidiert worden sein; wahrscheinlich war dann eine tödliche Meningitis die Folge. In der Mehrzahl der Fälle aber könnten Cephalhämatome behandelt worden sein. Selbst heute wird die Entleerung eines großen Cephalhämatoms durch Punktion oder Inzision noch hin und wieder durchgeführt. Wenn wir das Infektionsproblem ausklammern, das jeden chirurgischen Eingriff vor der Antibiotica-Ära belastete, sind die genauen Einzelheiten des Behandlungsplans, das im Prinzip bei den Autoren gleiche Vorgehen, genaue Angaben über das Behandlungskonzept (Heilung und Entleerung) und zur Heilungsdauer (Primärheilung am dritten Tag) starke Indizien dafür, daß die angegebenen Eingriffe tatsächlich - und erfolgreich - durchgeführt wurden. Eine Behandlung des Hydrocephalus in unserem Sinne ist damit allerdings in keinem Fall gemeint.

Wesentlich anders liegen die Verhältnisse bei der Behandlung der intrakraniellen Geschwülste. Hier differieren die Angaben beträchtlich. Pseudo-Galen schlägt, wie angegeben, die Trepanation bei den Ergüssen zwischen Knochen und Hirnhaut vor. Bei Aetios finden wir ebenfalls einen Behandlungsvorschlag, der jedoch ungleich kürzer, verschwommener und weniger nachvollziehbar ist als sein Therapiekonzept bei extrakraniellen Ergüssen. Es soll abgewartet werden, bis 
der Erguß die volle Größe erreicht hat; nach Nahtdehiszenz wird die Flüssigkeit sichtbar; dann solle man auf der Kuppe einschneiden und das «Angebrachte» tun. Den morphologischen Sachverhalt haben wir oben ausführlich erörtert. Wir wollen annehmen, daß mit der Kuppe der Flüssigkeitsgeschwulst die Vorwölbung der Fontanelle gemeint ist. Nach unseren Kenntnissen muß ein willkürlicher Einschnitt in diesem Bereich den Liquorraum eröffnet und in der Regel letale Folgen gehabt haben. Der Ausführung einer solchen Behandlung sollten also Grenzen gesetzt gewesen sein. Die Kürze der Beschreibung, das Fehlen jeglicher Einzelheiten der Schnittführung, der Verbände und des Heilverlaufs ist insbesondere im Vergleich zu den Angaben über die extrakraniellen Ergüsse auffällig.

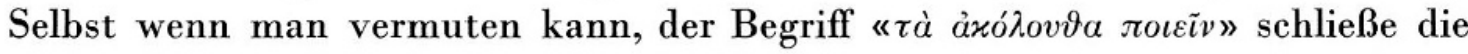
vorher gegebenen Beschreibungen ein und erspare so eine Wiederholung, so wäre doch die Ausführlichkeit der beschriebenen jeweiligen Behandlungsformen der extrakraniellen Geschwülste, die ja teilweise wörtlich Vorhergesagtes wiederholen, auffallend. Wir vermuten daher, daß der Beschreibung der Behandlung intrakranieller Ergüsse nur geringe oder keine praktische Erfahrung des Autors zugrunde liegt. Zumindest dürfte eine entsprechende Behandlung ohne Erfolg bzw. von katastrophalen Folgen begleitet gewesen sein. Wir haben bereits bei der Erörterung der Hydrocephalusform zwischen Hirnhaut und Hirn gesehen, daß vor allem bei Aetios noch keine exakte Abgrenzung seiner Auffassung von der früherer Autoren möglich war. Möglicherweise stoßen wir hier auf einen ähnlichen Zusammenhang. Frühere Autoren mögen die chirurgische Behandlung der intrakraniellen Geschwülste angegeben haben; Pseudo-Galen schlägt sie ohne nähere Erläuterung vor. Von Aetios bzw. seinem Quellenautor nehmen wir an, daß er diese Behandlungsmethode ohne eigene Erfahrung nachschrieb.

Eine völlig andere Auffassung finden wir bei Oreibasios und Paulos. Übereinstimmend lehnen beide jede chirurgische Behandlung des intrakraniellen Ergusses $a b$. Betrachten wir zunächst die Gründe bei Oreibasios. Wieder geht er von den bei ihm scharf getrennten Formen - Knochentumor oder Nahtdehiszenz mit Verlagerung der Flüssigkeitsgeschwulst - aus. Bei Nahtdehiszenz ist eine Heilung unmöglich, eine möglicherweise sehr mechanische Auffassung, bei der eine Überbrückung der großen Knochenlücken für unmöglich gehalten wird. Zudem gilt offenbar die Hirnhaut durch die Ergußflüssigkeit als so geschädigt, daß sich eine Therapie erübrigt. Interessant ist die Auseinandersetzung mit der Trepanationstherapie. Für Oreibasios ist die Deckung des Knochendefektes nach Entfernung des Knochentumors die Hauptschwierigkeit. Dafür gibt es keine Möglichkeit. Die Folge wäre, daß die Hirnhaut frei läge und der Kranke Krämpfe bekäme. Bereits im «Corpus Hippocraticum» («Über Schädelverletzungen») wird be- 
richtet, daß bei Verletzungen der Hirnhaut Krämpfe der kontralateralen Seite auftreten. Hier finden wir die gleiche Auffassung wieder. Vermutet werden kann, daß entweder Oreibasios bzw. sein Quellenautor selbst die Gefahr der Operationsmethode bei Knochentumoren erlebt hat, daß also z. B. nach Entfernung eines verkalkten Cephalhämatoms durch Trepanation Krämpfe aufgetreten sind oder daß andere Autoren bereits andere Vorschläge als beispielsweise Pseudo-Galen gemacht haben. Auf jeden Fall wird die operative Behandlung des intrakraniellen Ergusses abgelehnt. Ausdrücklich gegen die Auffassung der trepanierenden Chirurgen wendet sich auch Paulos. Leider gibt er keine Gründe für die Ablehnung an, so daß wir allerhöchstens spekulieren können, daß ähnliche Gründe wie bei Oreibasios entscheidend waren. Festgehalten werden muß auf jeden Fall, daß ganz im Gegensatz zur Behandlungspraxis der extrakraniellen Ergüsse die Skala der Behandlungsvorschläge bei den diagnostizierbaren intrakraniellen Prozessen von pauschaler Zustimmung bis zu kategorischer Ablehnung reicht. Wir möchten den Schluß ziehen, daß eine chirurgische Behandlung der extrakraniellen, von den antiken Autoren als Hydrocephalus bezeichneten Ergüsse möglich war und wohl erfolgreich durchgeführt wurde. Wenn dagegen ein Autor pauschal-vage Vorschläge macht für die Behandlung von Krankheitsbildern, in denen wir Beschreibungen eines Hydrocephalus in unserem Sinne wiedererkennen können, wenn dann bei anderen einhellig jede Behandlung gerade dieses Krankheitsbildes abgelehnt wird, dann scheint uns der Schluß erlaubt, daß, möglicherweise nach einer Phase «operativen Experimentierens», eine «realistische» Betrachtungsweise Platz gewann. Inwieweit möglicherweise die Ablehnung eines bei PseudoGalen aufgeführten Eingriffs auf einer kritischen Auseinandersetzung beruht, vermögen wir aufgrund allein dieser Texte nicht zu sagen. Wichtiger ist in unserem Zusammenhang die Feststellung, daß die begründete Ablehnung Praxisbezug zeigt.

Das aber erscheint uns, zurückkehrend an den Ausgangspunkt unserer Betrachtung, das Bemerkenswerteste: eine genaue Untersuchung der Texte deckt eine differenzierte Konzeption der Krankheitslehre und der Therapievorschläge auf. Vor diesem Hintergrund erscheint die Aussage, es gebe bereits in der Antike Behandlungsmöglichkeiten des kindlichen Hydrocephalus, nur dann über-

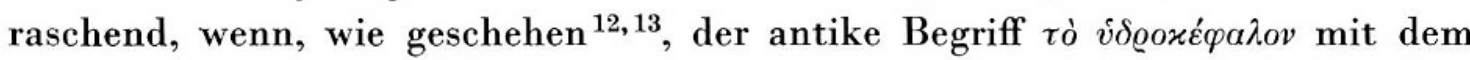
Hydrocephalus-Begriff der modernen Medizin gleichgesetzt wird. Wir haben zeigen können, daß der antike Begriff weitaus umfassender verstanden werden muß. Die, wie uns scheint, praktikablen, möglichen und wahrscheinlich ausgeführten Operationen betreffen dann aus unserer Sicht gänzlich andere Krankheitsbilder; für die antiken Autoren aber waren es Behandlungen des Hydro- 
cephalus in ihrem Sinne. Die wirklich utopische Behandlung, die operative Therapie des Hydrocephalus in unserem Sinne, aber wird in der Antike abgelehnt. Zumindest kann das für die spätere alexandrinische Chirurgie gesagt werden. Die Erkenntnis eines unzulänglichen, die Möglichkeiten weit übersteigenden Therapievorschlags, die Ablehnung einer utopischen Chirurgie auf einem bestimmten Sektor waren dann bereits medizinische Leistungen antiker Autoren.

\section{Anmerkungen}

1 Celsus, De medicina, Buch IV, 2. 3-9.

2 Galen, Werke. Ed. C.G.Kühn, Leipzig 1821/33, Bd.14, S.782, und Bd.19, S. 442 .

3 Pseudo-Soran, zitiert bei Gurlt, E., Geschichte der Chirurgie, Bd. I-III, Berlin 1898, Bd.I, S.407.

4 Aetios von Amida, Libri medicinales V-VIII, Ed. A.Olivieri, Nachdruck Berlin (Corp. Med. Graec. VIII 2, S.123-125).

5 Oreibasios, Collectionum medicarum reliquiae. Ed. J. Raeder, Nachdruck Amsterdam 1964 (Corp. Med. Graec. VI 2, S. 237-239).

6 Paulos von Aegina, Opera. Ed. J. C. Heiberg, Leipzig/Berlin 1924 (Corp. Med. Graec. IX 2, S.46-47).

7 a.a. O., S. 123, Z.5.

8 a.a.O., S. 46, Z.14.

9 Der edierte Text läßt die zuvor gegebene strenge Dreiteilung der Lokalisationsformen vermissen; die Lesart der Handschriften $\chi \mathrm{A}$ W für S.123, Zeile 21, erscheint uns darum sinnvoller, da sie die Gliederung der Schichten am Kopf in der schematischen Dreiteilung der Ergüsse wörtlich wiederholt.

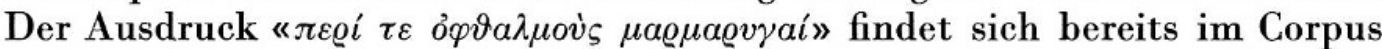
Hippocraticum. Es wird darunter eine ophthalmologische Symptomatik verstanden, die etwa dem "Sterne sehen» unseres Sprachgebrauchs entspricht, also mit blitzähnlichen Lichtsensationen einhergeht. Es fällt allerdings schwer, diese ganz auf subjektiver Schilderung beruhende Angabe auf kranke Neugeborene oder Kleinkinder zu beziehen. Zur Erklärung bietet sich an, daß der hippokratische terminus technicus in Verbindung mit häufigem Lidschlag gebraucht wird, bei Oreibasios also möglicherweise von diesem beobachtbaren Symptom aus auf die Lichtsensationen zurückgeschlossen wird. Nicht sehr wahrscheinlich, aber immerhin erwähnenswert,

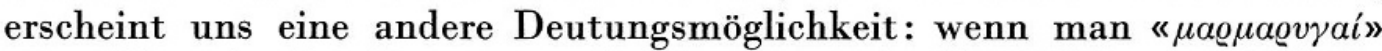
mit «blitzschnelles Bewegen» übersetzen würde (Liddell-Scott, $A$ GreekEnglish Lexicon, S.1081, Ausgabe 1973), dann könnten mit dem zur Rede stehenden Ausdruck möglicherweise sehr schnelle, nystagmusähnliche Augenbewegungen gemeint sein - eine Deutung, die gut zum klinischen Bild passen würde. 
11 Mackinney, L. C., and Herndon, Th. C., Abnormal cranial sutures in ancient, medieval and renaissance anatomical treatises, in: Studies in Honor of B. L. Ullman II, Roma 1964, S.413-426.

12 Gurlt, a.a. O., Bd. III, S. 644 ff.

13 Ghinopoulo, S., Pädiatrie in Hellas und Rom, Jena 1930, S.105ff.

\section{Summary}

Several ancient authors describe syndromes which they call themselves "hydrocephalus". So far it has been assumed that the "hydrocephalus" of these ancients is identical with our present day "hydrocephalus". Thus reports of operative treatment of hydrocephalus must surprise and sound utopian. A detailed analysis shows that the hydrocephalus notion of the ancients was broader and included diseases no longer regarded as hydrocephalus today. Comparison of passages shows that ancient authors were well aware of the utopian character of operating patients suffering from what we call hydrocephalus today, and limited their activities to extracranial collections of liquid, no longer called hydrocephalus today. The interpretation of the sources follows this evolution. From the data of the authors syndromes, corresponding to our present day definitions, are reconstructed. The therapeutics of the ancient authors then appear differentiated and realistic.

Dr. med. Ekkehard Pandel

D-23 Kiel

Freiligrathstraße 13 